play a crucial part in the effective management of treatments.

Severely mentally handicapped patients are usually unable to understand treatment or consent meaningfully to it. Few are formally detained and compelled to accept treatment. Mentally handicapped people usually acquiesce to what is done to them and they take the medication they are given.

The efficacy of psychiatric drugs is generally less certain and less predictable in mentally handicapped people, many of whom have existing evident or presumed brain damage or cerebral dysfunction. Some patients are sensitive to very small doses. Others tolerate amounts of antipsychotic drugs well in excess of British National Formulary standard dosage schedules.

In the community care of mentally handicapped people the role of the carer is interposed between doctor and patient. The doctor needs to gain the cooperation and confidence of the carers. The patient's compliance with treatment often depends on the carer's conforming with the doctor's advice and instructions. Carers, including parents and relatives, sometimes have their own attitudes, idiosyncratic views and prejudices. They do not necessarily follow strictly guidance about the administration of medicines. They cannot always be relied on to take care of medicines. Accidentally, if not deliberately, they can make changes from the intended drug regime. They may say only what they want the doctor to know.

The form of audit below summarises a checklist of points which arise in the drug treatment of mentally handicapped patients and needs to be recognised, reviewed and recorded.

A Form of Drug Audit in Mental Handicap Status of patient: informal/detained.

Consent: patient's capacity to understand and to consent to treatment medical treatment and second opinions

Place of drugs in overall treatment strategy and individual programme plan (IPP)

Appraisal of drug treatment:

-appropriate to psychiatric diagnosis

-appropriate to age and sex

-ability of patient to manage own self-medication and to care safely for-medicines; possible individual differences in metabolism of drugs

Drugs prescribed: anti-psychotic, anti-depressant, lithium, anti-epileptic, anti-parkinsonism, others, e.g. Cyproterone: dosage-low/normal/high; increasing/static/reducing; oral/tablets/liquid; depot injection

Combination of drugs: rationale, risk

Duration of therapy: days/weeks/months

Periodic monitoring and review: ward round, drug review meeting, case conference
Side effects: patient's ability to understand and to report side effects: none/slight/serious; observed/ complained of

Compatibility with other treatments: interaction with other drugs being taken

Blood plasma levels: lithium, anti-epileptics

Carers: involvement, attitudes, idiosyncratic views, understanding, willingness to care for medicines and handle their administration

Advice: information given to carer about medicines, e.g. handouts, patients' record cards.

Meanwood Park Hospital

D. A. SPENCER

Tongue Lane, Leeds LS6 4QD

\section{The role of physiotherapists in mental illness multidisciplinary teams}

\section{Dear Sirs}

I am prompted to write after reading the article 'Educating the Psychiatrist of the 21st Century' by R. H. Cawley (British Journal of Psychiatry, August $1990,174-181)$. His second educational theme covered mental health professionals and the psychiatric team, but did not mention physiotherapists. Our role is not fully appreciated, and there is a continual need to advertise our skills. We have been at pains to educate ourselves through a CSP validated course; we have a thriving special interest group, and regularly organise study days.

Physiotherapists are commonly thought of as being rehabilitators of the body rather than the mind. But there is a growing role for them as facilitators for the improvement of the mental health in conjunction with the physical health of their patients.

Patients suffering from mental illness are not exempt from physical problems. Our expectations and treatment plans are tempered by our knowledge and understanding of the patients' mental state and adjusted accordingly. We learn the value of interpersonal therapeutic relationships and counselling skills; attendance on ward rounds leads to an understanding of the roles of the other team members.

Physiotherapists are required to make an holistic assessment of the patient's problems and nowhere is this more apt than in the field of mental health, where body and mind are inseperable. We ask open questions and the nature of the answers indicates the patients' mental state.

Patients often blame their physical aches and pains for their mental state and will readily accept physical treatment because they believe it will make them better.

There is no stigma attached to seeing a physiotherapist. The practical way in which we assess problems and devise treatment programmes that encourage patients to utilise their own resources is 
acceptable to many people who would otherwise be reluctant to seek psychiatric help. The treatment programmes are a combination of traditional clinical methods and behaviour modification techniques or stress management skills, treating not just the presenting condition but the whole person.

Physiotherapists are also licensed to touch their patients. Touch evokes trust and caring. A neck and shoulder or back massage can produce deep relaxation and a sense of well being in the patient. Sharing problems with an active listener who is not there to give advice, but may occasionally direct the line of thinking so that the patient sees things for himself in a clearer light and forms his own solutions to his problems, can be the unlooked for bonus of a treatment session.

The patient who benefits most from a physiotherapeutic approach is the one with persistent symptoms that have devastatingly reduced his quality of life and for which no physical cause can be found. To be told, after lengthy investigations with a variety of consultants, that the problem is all "in the mind" is upsetting; to be summarily dismissed by a neurologist or an orthopaedic consultant and referred to a psychiatrist is the ultimate insult. Such patients finding themselves in a physiotherapy department, probably not for the first time, will naturally be sceptical. The key to a successful resolution lies in the approach to the problem. The focus of attention will be the quality of life. The patient identifies the areas of his life that he is dissatisfied with and wants to change. These are placed in order of priority and then worked at in small steps. This could include an exercise regime, learning stress management skills including relaxation, and planned graded activities. The reward and the motivation to continue what may be a long uphill struggle is the patient's recognition of his achievements. The therapist does not hide the fact that the patient should expect to work hard, and it will be painful and that there may be relapses. Ultimately the patient has a choice. He can improve the quality of his life in spite of his symptoms, or he can continue his search for a diagnosis that would absolve him from any responsibility for his condition. Experience has shown me that those who choose the former are grateful not only to be released from the prison of their pain but also for a therapeutic relationship that allows them to maintain their dignity. The therapist is the catalyst to a successful outcome and not the crutch that the patient learns to lean on.

There is also a role for the physiotherapist in the treatment of disorders such as anorexia nervosa that have a body image component. Using the non-verbal skills of massage, relaxation and movement, it is possible to facilitate an internal as well as an external feeling of self awareness. This, combined with practical stress management coping skills, induces a greater sense of acceptance and understanding of the body while the patient is undergoing a treatment package of weight restoration followed by psychotherapy. It will be appreciated that this is a perhaps surprisingly subtle way of applying generic physiotherapy skills to a specific condition. It is a far cry from the "jolly hockey sticks" approach for which we are sometimes known and which has a very limited role in psychiatry.

Physiotherapists working on multidisciplinary substance abuse rehabilitation teams, encourage their clients to be actively involved in their own physical rehabilitation. They organise circuit training sessions and take and direct clients to leisure activities. They take an active counselling role in health education and stress management programmes. As these clients tend to be "head" orientated people who blot out their problems and anxieties by eliminating them or dulling them through the abuse of alcohol or drugs, the awareness of a body that feels stiff after exercise, helps bring them in touch with their whole self. So exercise has the dual function of improving fitness and integrating head and body into a whole person.

It would not be right to conclude without mentioning the valuable work that we do with the elderly, working alongside the nurses to maintain patients' functional mobility and independence; or with the long-stay in- and out-patients whose smoke-abused chests and poor posture we endeavour to improve.

So I would agree with Professor Cawley that "properly arranged team work is a powerful way of helping the mentally disordered person, it is a most profitable modus operandi for the workers, and it is absolutely necessary for the satisfactory education of psychiatrists". I would also make a special plea that psychiatrists in the course of their training learn how the skills of the physiotherapist can benefit their patients.

Supt Physiotherapist, Mental Health Unit Coney Hill Hospital, Gloucester GL4 7QJ

\section{Abuse of mentally handicapped adults}

\section{DeAR Sirs}

I read with much interest and relief the article on 'Abuse of Mentally Handicapped Adults' by Leila Cooke (Psychiatric Bulletin, October 1990, 14, 608-609). It is an issue that has only recently been acknowledged by those working in the field, and yet we know from our own clinical experience that this aspect of 'care' has been a silent one. Little research has been done in this difficult area, but we do have pointers to its prevalence, knowing the heightened vulnerability of our patient/client group.

In my own clinical practice I know of mentally handicapped adults who have been sexually abused in 